\title{
Effet de la texture et du potentiel hydrique des sols sur l'expression du charbon des inflorescences du maïs (Graminées, Poaceae)
}

\author{
A. Teferi, M. Petitprez, V. Valles et L. Albertini \\ ENS Agronomique ,145 avenue du Muret, 31076 Toulouse Cedex, France \\ (reçu le 30 août 1988, accepté le 18 avril 1989)
}

\begin{abstract}
Résumé - Cinq types de sols très différents, dont le taux d'argile varie entre $8,1 \%$ et $25 \%$, contaminés artificiellement par des téliospores de Sphacelotheca reiliana, ont été maintenus à trois niveaux de potentiel hydrique. L'étude de la sévérité de la maladie montre qu'elle s'exprime en fonction inverse de la disponibilité du sol en eau. II n'y a pas d'influence notable du type de sol, dont la texture semble intervenir uniquement par le biais du potentiel hydrique, en permettant une plus ou moins grande disponibilité en eau.
\end{abstract}

potentiel matriciel - oxygénation - sévérité - Sphacelotheca reiliana

Summary - Influence of soil water potential and soil texture on infection of maize by head smut. Five different types of soil differing by their clay rate (8.1\% to 25\%) inoculated with spores of Sphacelotheca reiliana were adjusted to 3 levels of water potential. The study of disease severity shows that more maize seedlings were smutted at low matric potentials than at high ones. There is no appreciable effect of soil type, which seems to interfere on disease expression only through water potential, allowing plants to dispose of more or less water.

matric potential - oxygen potential - disease severity - Sphacelotheca reiliana

\section{INTRODUCTION}

Le charbon des inflorescences du maïs, dû à Sphacelotheca reiliana, est une maladie d'origine tellurique. Le maïs est sensible depuis la germination jusqu'au stade 7 feuilles (Zhang, 1982). Le mycélium se développe ensuite de façon systématique, et envahit les méristèmes floraux, provoquant la formation de galles charbonnées.

Plusieurs études (Christensen, 1926; Kruger, 1962) ont montré que certains facteurs abiotiques sont susceptibles d'influencer le développement de la maladie, notamment l'état physicochimique du sol et son humidité. Les observations de terrain relient la présence de la maladie soit à des sols légers (Kruger, 1962; Matyac \& Khommedahl, 1985), soit à des sols lourds présentant des zones d'hydromorphie (Flamant, 1985). Ces observations pouvant paraître contradictoires, nous nous sommes pro- posés de voir si la texture du sol et sa disponibilité en eau ont une influence sur la contamination de jeunes plants de maïs. Ayant préalablement montré l'existence d'une relation étroite entre la sévérité de la maladie et le nombre de téliospores présentes dans le sol (Teferi \& Petitprez, 1987), nous avons cultivé du maïs en conditions contrôlées jusqu'au stade 5-6 feuilles sur différents sols artificiellement contaminés et maintenus à un potentiel hydrique déterminé.

\section{MATÉRIEL ET MÉTHODES}

Les 5 échantillons de sol ont été choisis comme représentatifs des sols du Sud-Ouest de la France: 2 bouldènes (sols lessivés hydromorphes) ( $n^{\circ} 1$ et 2 ), 1 sable fauve acide $\left(n^{\circ} 3\right), 1$ terrefort (sol brun calcaire, argileux) $\left(n^{\circ} 4\right), 1$ limon argilo-sableux $\left(n^{\circ} 5\right)$. Les prélèvements ont été effectués entre 5 et $20 \mathrm{~cm}$ de profondeur et leurs caractéristiques sont résumées dans le Tableau I. Ces échantillons ont été désinfectés à la 
Tableau I. Paramètres texturaux des différents sols utilisés (en g par kg de sol sec).

Argile CaO Sable Limon M.O. $\mathrm{pH}$

\begin{tabular}{|c|c|c|c|c|c|c|}
\hline 1. Boulbène & 194 & 0 & 216 & 384 & 19 & 6,9 \\
\hline 2. Boulbène & 233 & 5,0 & 250 & 344 & 26 & 7,5 \\
\hline $\begin{array}{l}\text { 3. Sable fauve } \\
\text { acide }\end{array}$ & 81 & 0 & 121 & 102 & 14,7 & 5,10 \\
\hline 4. Terrefort & 243 & 287 & 197 & 226 & 16 & 8,50 \\
\hline $\begin{array}{l}\text { 5. Limon argilo- } \\
\text { sableux }\end{array}$ & 251 & 11 & 200 & 345 & 35 & 7,30 \\
\hline
\end{tabular}

chaleur $\left(60 \min\right.$ à $\left.100^{\circ} \mathrm{C}\right)$, contaminés de manière homogène par brassage de 1 volume de téliospores pour 200 volumes de sol, soit 1,1.106 téliospores. ${ }^{-1}$, puis répartis dans des pots de 12 l, dans lesquels nous avons semé une lignée de maïs sensible au Sphacelotheca reiliana. Le dispositif choisi est un essai en randomisation totale avec répétitions, comportant 30 plantes par type de sol pour un potentiel hydrique donné.

Le potentiel hydrique (Bourrie, 1983) est proportionnel à l'énergie minimale que doit fournir un organisme pour extraire de l'eau du sol. II détermine la disponibilité énergétique de l'eau. Ce potentiel est mesuré par un tensiomètre à mercure, dispositif fiable et précis, notamment en conditions isothermes (Bruckler, 1984). Les tensiomètres, construits à partir de bougies poreuses Nardeux de $32 \mathrm{~mm}$ de long et $12 \mathrm{~mm}$ de diamètre, ont une surface de contact avec le sol suffisante et donc un temps de réaction très court (quelques dizaines de s). lls sont installés à $10 \mathrm{~cm}$ de profondeur et reliés à un réservoir de mercure par un tube déployé sur une règle graduée en $\mathrm{cm}$ d'eau $(1 \mathrm{~cm}$ d'eau $=-10^{2} \mathrm{~Pa}$ ). Le potentiel hydrique peut également être exprimé en pF, c'est-à-dire en logarithme décimal de la valeur lue sur la réglette. Un potentiel hydrique faible, c'est-à-dire un pF élevé, traduit une difficulté d'alimentation hydrique de la plante. Nous avons, par des arrosages précis, maintenu les potentiels hydriques des sols autour de trois paliers:

- de $-4,7.10^{4} \mathrm{~Pa}$ à $-2,10^{4} \mathrm{~Pa}$, soit un $\mathrm{pF}$ de 2,67 à 2,30 (valeur moyenne $-3,2.10^{4} \mathrm{~Pa}$ )

- de $-4,0.10^{4} \mathrm{~Pa}$ à $-1,0.10^{4} \mathrm{~Pa}$, soit un $\mathrm{pF}$ de 2,60 à 2 (valeur moyenne $-2,5.10^{4} \mathrm{~Pa}$ )

- de $-1,0.10^{4} \mathrm{~Pa}$ à $-1,0.10^{3} \mathrm{~Pa}$, soit un $\mathrm{pF}$ de 2 à 1 (valeur moyenne $-4,0.10^{3} \mathrm{~Pa}$ )

Ces valeurs correspondent aux bornes entre lesquelles le potentiel a varié au cours de l'expérimentation. L'analyse de variance des mesures quotidiennes montre que les trois traitements constituent des niveaux significativement distincts.

Au centre de chaque pot, nous avons disposé un tube percé dans sa moitié supérieure, afin de collecter l'eau de percolation. La teneur en oxygène dissous dans cette eau est mesurée quotidiennement, grâce à un oxymètre Yellow Spring Inc. ; modèle 57. Cette valeur est proportionnelle à la pression partielle en oxygène $\left(\mathrm{pO}_{2}\right)$ équilibrant la solution du sol: $\mathrm{O}_{2}$ dissous $=10^{2.87} \times \mathrm{pO}_{2}$ (Fritz, 1981). Nous l'exprimerons en pourcentage par rapport à la teneur en oxygène dissous sous 1 atmosphère. A titre d'exemple, un sol bien aéré possède une pression en oxygène comprise entre $18 \%$ et $20,5 \%$.

Pendant les quatre semaines de l'expérimentation en conditions contrôlées, les plantes sont maintenues en chambre de culture: $12 \mathrm{~h}$ d'illumination à $100 \mu \mathrm{E} \cdot \mathrm{m}^{-2} \cdot \mathrm{s}^{-1}$ à $30^{\circ} \mathrm{C}$ et $60 \%$ d'humidité relative, et $12 \mathrm{~h}$ d'obscurité à $26^{\circ} \mathrm{C}$ et $80 \%$ d'humidité. Parvenus au stade 6 feuilles, les plants de maïs sont transférés en conditions naturelles jusqu'à maturité. Nous quantifions alors l'effet du parasite en estimant la sévérité de la maladie sur la plante entière, selon la grille de notation (Tableau II). La sévérité est exprimée par la somme de trois notes partielles portant, la première sur l'inflorescence mâle (de 0 à 6 ), la seconde sur l'épi (de 0 à 6), la troisième sur l'aspect général de la plante (de 0 à 2); la note globale varie donc de 0 à 14 . Ce système de notation de la sévérité présente une bonne corrélation avec la densité de l'inoculum du sol (Teferi \& Petitprez, 1987).

Tableau II. Grille de notation de la sévérité $(S=P+E$ + T).

\begin{tabular}{llllll}
\hline \multirow{2}{*}{ Panicule } & \multicolumn{2}{c}{ Epi } & \multicolumn{3}{c}{ Aspect de la plante } \\
Symptômes & $P$ & Symptômes & E & Taille & $T$ \\
\hline Saine & 0 & Sain & 0 & Normale & 0 \\
Modifiée & 2 & Modifiée & 2 & Réduite & 1 \\
Phyllodiée & 4 & Phyllodié & 4 & Plante naine & 2 \\
Charbonnée & 6 & Charbonnée & 6 & & \\
& & & &
\end{tabular}

\section{RESULTATS}

\section{Potentiel hydrique}

L'utilisation du tensiomètre à bougie pour la mesure du potentiel hydrique s'est révélée satisfaisante pour la gamme de disponibilité en eau que nous nous étions fixée: potentiel matriciel inférieur, en valeur absolue, à $0,8.10^{5} \mathrm{~Pa}$. Les résultats du Tableau III montrent une bonne relation entre le potentiel hydrique et la sévérité de la maladie: au niveau élevé de disponibilité en eau $\left(-4.10^{3} \mathrm{~Pa}\right)$ sont associées les plus faibles sévérités observées; inversement, au faible potentiel hydrique $\left(-32.10^{3} \mathrm{~Pa}\right)$, nous trouvons les plus fortes sévérités. La comparaison des différents sols entre eux fait ressortir une grande homogénéité de réaction; à l'exception du limon argilosableux $n^{\circ} 5$, les sévérités ne varient pas significativement en fonction du type de sol, mais uniquement en fonction du potentiel hydrique appliqué. Ces résultats indiquent l'existence d'une relation inverse entre l'expression de la maladie et l'eau disponible dans le sol.

\section{Oxygénation du sol}

Les problèmes d'aération, liés à la disparition de l'air de la porosité, soit un indice de l'air nul, sur- 
viennent habituellement pour des potentiels matriciels voisins de zéro (Tessier, 1984). Cependant, dans la gamme de pF choisie (Tableau IV), on constate que l'oxygénation de l'eau du sol est importante, et ne varie pas selon le sol.

\section{DISCUSSION}

\section{Influence du type de sol}

Les cinq sols que nous avons étudiés présentent des caractéristiques fort différentes. Malgré ces différences importantes de texture, nous n'avons pas pu mettre en évidence de différence de sen-

Tableau III. Sévérité associée aux cinq types de sol maintenus à différents potentiels hydriques. Les lettres représentent les groupes homogènes de sévérité, d'après le test de Newman \& Keuls (Dagnelie, 1975).

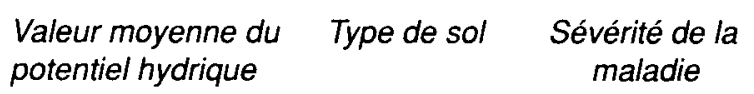

\begin{tabular}{llll}
\hline & & & \\
& 1 boulbène & 6,0 & $\mathrm{a}$ \\
& 2 boulbène & 5,37 & $\mathrm{a}$ \\
$-4,0.10^{3} \mathrm{~Pa}$ & 3 sable fauve & 5,25 & $\mathrm{a}$ \\
& 4 terrefort & 5,37 & $\mathrm{a}$ \\
& 5 limon & 4,62 & $\mathrm{a}$ \\
& & & \\
& 1 boulbène & 9,37 & $\mathrm{~b}$ \\
& 2 boulbène & 8,50 & $\mathrm{~b}$ \\
& 3 sable fauve & 8,87 & $\mathrm{~b}$ \\
& 4 terrefort & 9,12 & $\mathrm{~b}$ \\
& 5 limon & 8,5 & $\mathrm{~b}$ \\
& & & \\
& 1 boulbène & 12,0 & $\mathrm{c}$ \\
& 2 boulbène & 11,75 & $\mathrm{c}$ \\
& 3 sable fauve & 11,25 & $\mathrm{c}$ \\
$-32.10^{3} \mathrm{~Pa}$ & 4 terrefort & 12,62 & $\mathrm{c}$ \\
& 5 limon & 9,37 & $\mathrm{~b}$ \\
\hline & & & \\
& & &
\end{tabular}

Tableau IV. Pourcentage d'oxygène dans les solutions de percolation, pour un potentiel hydrique de $-4.10^{3} \mathrm{~Pa}$. Les lettres désignent les groupes homogènes d'après le test de Newman \& Keuls (Dagnelie 1975).

\section{Type de sol}

Oxygène en \%

\begin{tabular}{lll}
1 boulbène & 14,56 & a \\
2 boulbène & 14,80 & a \\
3 sable fauve & 14,10 & a \\
4 terrefort & 15,30 & a \\
5 limon & 14,90 & a \\
\hline
\end{tabular}

sibilité du maïs au Sphacelotheca reiliana liée au type de sol. Ces résultats vont à l'encontre des travaux de Kruger (1962), qui, ayant obtenu deux fois moins d'infection sur sol argileux que sur sol sableux, conclut que la texture du sol jouerait un rôle important dans le développement de Sphacelotheca reiliana. Cette contradiction n'est peutêtre qu'apparente et liée à la différence des paramètres expérimentaux contrôlés: Kruger a effectué des observations sur deux types de sol, sans tenir compte de leurs différentes capacités de rétention d'eau. Dans notre investigation, les différences de texture n'influent plus sur la disponibilité en eau, son accessibilité est identique quel que soit le type de sol, pour un potentiel hydrique donné. Considérées de cette manière, les observations de Kruger (1962) viendraient corroborer l'effet antagoniste qu'exerce l'eau d'un sol sur le développement de la maladie.

\section{Potentiel hydrique}

Selon le niveau de disponibilité en eau du sol, le Sphacelotheca reiliana occasionne des dégâts plus ou moins importants: la maladie s'exprime d'autant moins que le potentiel hydrique du sol est élevé. Ce résultat vient confirmer les travaux de Matyac \& Kommedahl (1985), qui trouvent que les températures élevées et les faibles potentiels matriciels favorisent le charbon des inflorescences. De même, à partir d'observations faites au champ, Christensen (1926) et Kruger (1962) rapportent que le maïs est peu infecté s'il est semé après et au cours de chutes régulières de pluie; selon Kruger, la quantité totale de pluie, et donc l'humidité du sol, seraient susceptibles de moduler l'expression de la maladie. En pratiquant des irrigations fréquentes, Mack et al. (1984) notent qu'un apport de 15 à $20 \mathrm{~cm}$ d'eau durant les trois semaines suivant le semis permet de réduire l'incidence du Sphacelotheca reiliana sur le maïs. Tout ceci montre donc que le potentiel hydrique joue un rôle déterminant dans l'expression de la maladie.

Deux hypothèses explicatives peuvent être invoquées: soit les faibles potentiels matriciels affaiblissent la plante, la prédisposant ainsi à la colonisation par le parasite, soit, dans les sols gorgés d'eau, la faible disponibilité en oxygène est susceptible de freiner l'activité du Sphacelotheca reiliana.

\section{Oxygénation du sol}

L'oxygénation du sol est un domaine mal connu, du fait de problèmes théoriques et expérimen- 
taux de définition des normes. Dans notre expérimentation, la quantité d'oxygène mesurée est celle dissoute dans l'eau qui a percolé par la porosité grossière, donc dans les parties les plus aérées du sol. Selon Levy \& Toutain (1979), dans un sol bien aéré, l'oxygène représente 18 à $20 \%$ de la phase gazeuse du sol; après une pluie, ce pourcentage peut descendre à $10 \%$ et jusqu'à $2 \%$ dans des cas de mauvaise structure. Comparée à ces valeurs, la teneur moyenne de nos sols, 14 à $15 \%$, refléterait une aération convenable, même au potentiel hydrique le plus élevé. L'oxygène disponible ne semble pas être un facteur modulant le développement de la maladie.

\section{Sensibilité et stress hydrique}

Nos résultats indiquent que moins le maïs dispose d'eau, plus il devient sensible au Sphacelotheca reiliana. Pour les valeurs faibles du potentiel hydrique $\left(-32.10^{3} \mathrm{~Pa}\right)$ que nous avons testées, nous nous situons dans des conditions de sécheresse qui, pour de jeunes plants de maïs, avoisinent le déficit hydrique (Barloy, 1983). Si le maïs tolère des niveaux d'humidité élevés, et même des sols peu ou mal aérés (Grobbelaar, 1981, cité par Bonhomme, 1983), il est par contre très sensible aux déficits d'alimentation hydrique (Derieux, 1983).

Or, pour de nombreux auteurs ayant étudié les incidences de la disponibilité en eaux sur le développement de maladies racinaires (Leviti, 1980, cité par Schoeneweiss, 1986; Hall, 1986; Hanson \& Hitz, 1982), il existerait un phénomène d'affaiblissement de la plante sous l'effet d'un stress hydrique. Ce stress peut être causé par un déficit pluviométrique, par une restriction de la croissance racinaire, ou par des perturbations de la prise d'eau et de son transport dans la plante (Waller, 1986). Dans notre expérimentation, les faibles niveaux de potentiel hydrique constituent des conditions d'autant plus drastiques que la culture en pot ne permet à la plante que de disposer d'un volume réduit de sol à prospecter. L'enquête menée par Flamant (1985) auprès de maïsiculteurs du Sud-Ouest a montré que le charbon des inflorescences était souvent présent en bordure de parcelles, sur les zones tassées et les zones de mouillères. Ces localisations correspondent à des zones présentant des risques d'hydromorphie entraînant un mauvais développement du système racinaire du maïs. La plante, résistant mal a la sécheresse, serait plus sensible à la maladie. II y aurait donc un phénomène de prédisposition au charbon des inflorescences de maïs soumis à des conditions de stress hydrique.

\section{CONCLUSION}

La capacité des sols très humides à diminuer la sévérité des attaques du charbon des inflorescences du maïs ne peut pas s'expliquer par une limitation de l'activité du parasite par anoxie, puisque, même à des potentiels hydriques très élevés, l'oxygène disponible, représentant plus de $14 \%$, reflète un sol convenablement aéré. On peut donc penser que, pour de faibles disponibilités en eau, le déficit hydrique subi par la plante affaiblirait ses mécanismes de défense, la prédisposant ainsi à la maladie. Une meilleure connaissance des réactions de défense du maïs contre Sphacelotheca reiliana permettrait de comprendre le lien existant entre le stress hydrique et la prédisposition au charbon des influorescences.

\section{REMERCIEMENTS}

Ce travail a été réalisé dans le cadre d'une collaboration avec Pioneer-France Maïs.

\section{RÉFÉRENCES}

Barloy J. (1983) Phase de germination, levée et implantation. In: Physiologie du Maïs, Colloque INRA. 13-48

Bonhomme R. (1983) Mise en place des appareils foliaire et racinaire. In: Physiologie du Maïs, Colloque INRA, 63-85

Bourrie G. (1983) Potentiel osmotique et potentiel matriciel. Communication au GFHN Rennes, 14, 39-46

Bruckler L. (1984) Utilisation de la tensiométrie en situation non isotherme. Sci. Sol. 1, 5-21

Christensen J.J. (1926) The relation of soil temperature and soil moisture to development of head smut in sorghum. Phytopathology 16, 353-357

Dagnelie P. (1975) Théorie et Méthodes Statistiques, vol. 2. Les Méthodes de I'Inférence Statistique. La Presse Agronomique de Gembloux, 2e éd.

Derieux M. (1983) Les principaux facteurs limitants du milieu, In: Physiologie du Maïs, Colloque INRA. 383387

Flamant P. (1985) Le charbon des inflorescences du maïs. Mémoire de fin d'études, ESAP, Toulouse

Fritz B. (1981) Etude thermodynamique et modélisation des réactions hydrothermales et diagénétiques. Sci. Géol. Mém. 65

Hall R. (1986) Effect of root pathogens on plant water relations. In Water, Fungi and Plants. Br. Mycol. Soc. Sympos. 11, 119-137

Hanson A.D. \& Hitz W.D. (1982) Metabolic response of mesophytes to plant water deficits. Annu. Rev. Plant Physiol. 33, 163-203

Kruger W. (1962) Sphacelotheca reiliana on maize; infection and control studies. S. Afr. J. Agric. Sci. 5, 43-56 
Levy \& Toutain F. (1979) Aération et phénomènes d'oxydoréduction dans le sol. In: Pédologie, 2 vol. (M. Bonneau \& B. Souchier eds.) Masson, Paris

Mack H.J., Bugget J.R. \& Koepsell P.R. (1984) Effects of cultural practices on the incidence of head smut in sweet corn. Hortic. Sci. 19, (1), 77-78

Matyac C.A. \& Kommedahl T. (1985) Factors affecting the development of head smut caused by Sphacelotheca reiliana on corn. Phytopathology 75, 577-581

Schoeneweiss D.F. (1986) Water-stress predisposition to disease an overview. In: Water, Fungi and Plants. Br. Mycol. Soc. Symp.11, 157-174

Teferi A. \& Petitprez M. (1987) Mise au point d'une méthode de détection des spores de Sphacelotheca reiliana et étude de l'influence de la densité d'inoculum sur la sévérité de la maladie. fer Cong. S.F.P. Rennes

Tessier D. (1984) Etude expérimentale de l'organisation des matériaux argileux: hydratation, gonflement et structuration au cours de la dessiccation et de la réhumectation. Thèse Doct. ès Sci. Paris VII

Waller J.M. (1986) Drought, irrigation and fungal diseases of tropical crops in: Water, Fungi and Plants. Br. Mycol. Soc. Sympos. 11 175-187

Zhang Z. (1982) On the disease incidence of maize head smut. (Sphacelotheca reiliana). Acta Phytopathologica Sin. 12, 33-40 